\title{
Conception actuelle du traitement de la côlite ulcéreuse
}

La formule de «panel discussion» présente deux avantages essen-tiels sur les rapports exposes successivement, puis suivis de communications: elle oblige les rapporteurs à une très grande concision - il s'agit d'introduire la question, non de $\Gamma$ exposer - et en permet la discussion immediate. Les organisateurs hollandais du recent Congrès européen de gastro-entérologie [Leyden et Noordwíjk, avril 1960) avaient choisi cette manière de traiter les sujets à $\Gamma$ ordre du jour, ce qui nous autorise à formuler des conclusions générales au lieu de $\Gamma$ opinion de quelques conférenciers.

La panel discussion sur la côlite ulcéreuse avait été préparée minu-tieusement par S. Truelove, d'Oxford, qui sut en diriger les débats de façon fructueuse.

Dans les formes graves aíguës de la maladie, chacun s'accorde à con-trôler Гéquilibre liquidien et électrolytique, à transfuser selon lebesoin, et à combattre les carences nutritionnelles et vitaminiques. Parmi les medicaments spécifiques, de nombreux médecins restent fídèles à la Salazopyríne, préconisée par Mme Nanna Svartz (Stockholm), et rendent hommage aux services rendus par les cortícostéroïdes; G. Wat-

\section{Editorial}

323

kínson (Leeds) estime ГA.C.T.H. supérieur et pretend obtenir des ré-sultats equivalents à ceux publiés en Amérique avec des doses moitié moindres. Les désinfectants sont nécessaires pour combattre la sur-infection, mais les antíbiotiques à large spectre d'action ont parfois provoqué des catastrophes, et doivent être prescrits avec circonspec-tion et de preference par voie veineuse.

Les chirurgiens sont très optimistes sur leurs possibilités, même dans les formes suraiguës et St. Aylett (Londres) affirme n'avoir jamais refuse d'intervenir à cause d'un état general trop précaire! C'est pour ces états gravissimes que JR. Cattan (Paris) se fait l'avocat de Vopéra-tíon de Bucaílle (infiltration novocaïnique limitée d'une zone de substance blanche préfrontale) qui ne pretend pas guérir la côlite ulcé-reuse, ni éviter une côlectomie secondaire, mais sauver des cas désespé-rés - surtout en presence d'hémorragies profuses.

Dans les formes chroniques à rechutes, outre les moyens utilises dans les formes aiguës - les corticostéroïdes agissant bien contre les pous-sées évolutives, mais ne modifiant guère le cours general de la maladie, et ne préviennent pas Гapparition de rechutes - on est arnené, comme dans les formes chroniques continues, à mettre en train d'autres mé-thodes.

La psychothérapíe ne saurait être «profonde» (analyse, etc.), car elle risque alors de faire plus de mal que de bien, et doit rester tech-niquement «superficielle» (Groen, Jerusalem). II faut surtout montrer à ces malades de Гintérêt, avoir avec eux une patience infinie, les encourager et les entourer ... bref, un type de psychothérapie plus proche de celle du médecin-praticien - quand il peut y consacrer assez de temps - que de celle du spécialiste.

II en est de même des soins médicaux persévérants, capables d'amé-liorer - on n'ose jamais parler de guérison avec cette maladie - la grande majorité de ces ulcéreux. Encore leur faut-il une alimentation appropriée: une ration hypercaloríque hyperprotéínée s'impose (M. De-mole, 
Geneve), qui n'a pas besoin d'etre privée de cellulose (fruits et legumes) quand les lesions siègent seulement sur les colons descendant et terminal. Plus récemment enfin, on a soulevé Thypothèse d'une cause allergíque alímentaíre à la côlite ulcéreuse.

La nécessité d'un traítement chirurgícal est diversement appréciée, et celui-ci semble plus souvent nécessaire dans les pays du Nord que dans l'Europe occidentale ou méditerranéenne, où il ne dépasse pas $10 \%$ des cas. La côlectomie totale ou subtotale reste Topération de choix, mais avec Vanastomose íléo-rectale, St. Aylett évite aux malades 